OPEN ACCESS

Edited by:

Alessia Passanisi, Kore University of Enna, Italy

Reviewed by:

Ziqing Deng,

Beijing Genomics Institute (BGl), China

Jianming Zhu,

Second Affiliated Hospital of

Nanchang University, China

*Correspondence:

Anwen Shao

shaoanwen@zju.edu.cn

Min Zheng

minz@zju.edu.cn

Specialty section:

This article was submitted to

Psychopathology,

a section of the journal

Frontiers in Psychiatry

Received: 01 January 2021

Accepted: 26 April 2021

Published: 19 May 2021

Citation:

Qian H, Ling Y, Wang C, Lenahan C,

Zhang $M$, Zheng $M$ and Shao $A$ (2021) A Correlative Study Between Personality Traits and the Preference

of Site Selection in Cosmetic

Treatment.

Front. Psychiatry 12:648751.

doi: 10.3389/fpsyt.2021.648751

\section{A Correlative Study Between Personality Traits and the Preference of Site Selection in Cosmetic Treatment}

\author{
Huan Qian ${ }^{1}$, Yuxiao Ling ${ }^{2}$, Chen Wang ${ }^{1}$, Cameron Lenahan ${ }^{3,4}$, Mengwen Zhang ${ }^{1}$, \\ Min Zheng ${ }^{5 *}$ and Anwen Shao ${ }^{6 *}$ \\ ${ }^{1}$ Department of Plastic Surgery, The Second Affiliated Hospital, School of Medicine, Zhejiang University, Hangzhou, China, \\ ${ }^{2}$ School of Public Health, Hangzhou Medical College, Hangzhou, China, ${ }^{3}$ Center for Neuroscience Research, Loma Linda \\ University School of Medicine, Loma Linda, CA, United States, ${ }^{4}$ Burrell College of Osteopathic Medicine, Las Cruces, NM, \\ United States, ${ }^{5}$ Department of Dermatology, The Second Affiliated Hospital, School of Medicine, Zhejiang University, \\ Hangzhou, China, ${ }^{6}$ Department of Neurosurgery, The Second Affiliated Hospital, School of Medicine, Zhejiang University, \\ Hangzhou, China
}

Background: Cosmetic treatment was closely associated with beauty seekers' psychological well-being. Patients who seek cosmetic surgery often show anxiety. Nevertheless, not much is known regarding how personality traits relate to the selection of body parts that receive cosmetic treatment.

Aims: This study aims to investigate the correlation between personality traits and various selection sites for cosmetic treatment via Eysenck Personality Questionnaire (EPQ).

Methods: A cross-sectional approach was adopted to randomly recruited patients from a general hospital planning to undergo cosmetic treatments. All respondents completed the EPQ and provided their demographic information. The EPQ involves four scales: the extraversion $(E)$, neuroticism $(N)$, psychoticism $(P)$, and lying scales $(L)$. Psychological scales were evaluated to verify that people who selected different body sites for cosmetic intervention possessed different personality portraits.

Results: A total of 426 patients with an average age of $32.14 \pm 8.06$ were enrolled. Among them, 384 were females, accounting for more than $90 \%$ of patients. Five treatment sites were analyzed, including the body, eye, face contour, nose, and skin. Comparatively, patients with neuroticism were more likely to undergo and demand rhinoplasty (OR 1.15, 95\% Cl 1.07-1.24, $P<0.001)$. Face contour treatment was commonly associated with extraversion (OR 1.05, 95\% Cl 1.00-1.11, $P=0.044$ ), psychoticism (OR 1.13, Cl 1.03-1.25, $P=0.013$ ), and neuroticism (OR 1.05, Cl 1.01-1.10, $P=0.019$ ).

Conclusions: This novel study attempted to determine the personality profiles of beauty seekers. The corresponding assessments may provide references for clinical treatment options and enhance postoperative satisfaction for both practitioners and patients.

Keywords: personality traits, cosmetic, personality questionnaire, rating scale, surgery 


\section{INTRODUCTION}

The pursuit of physical beauty is on the rise (1). Cosmetic treatment refers to modifications made to the human body's appearance in the absence of disease, injury, wound, congenital, or hereditary deformity, while also improving the quality of life (2). According to the American Society for Aesthetic Plastic Surgery (ASAPS), Americans splurged over $\$ 15$ billion on cosmetic procedures in 2016 (3). In 2018, the total number of surgical and non-surgical procedures surged to 21.5 and 30.5\%, respectively, over the last 5 years (4). The International Society of Aesthetic Plastic Surgery (ISAPS) ranked China second in this specific growth trend (5). As the demand for aesthetic procedures grows, people wish to transform their physical appearance to improve their psychological profile and psychosocial wellbeing (6).

People undergoing plastic surgery are not traditional patients with physical health issues. Psychological factors greatly motivate them to pursue surgery. Previous studies pertaining to aesthetic psychology mainly focused on cosmetic patients suffering from mental illnesses. Body dysmorphic disorder (BDD) is among the three most common psychiatric disorders in cosmetic patients (7). BDD patients are especially sensitive to minor flaws in their appearance (8) and demonstrate a tendency for dissatisfaction with surgical outcomes (9). Another common observation by past studies is borderline personality disorder (BPD), which was linked to emotional imbalance, impulsiveness, and self-image issues (10). These patients relentlessly demand and seek cosmetic surgery for self-injury. Hence, surgical treatment is best avoided in these patients (11).

Personality disorders are founded on the scientific principles of personality traits (12). However, the normal personality profiles in an average population are underreported. Here, some personality aspects of the general population seeking cosmetic treatments are analyzed. Various reports were linked with neuroticism and negative personality aspects; neuroticism is the most common personality trait in rhinoplasty patients (13). Perception of attractiveness increase in these rhinoplasty patients, improving their evaluation of their own attractiveness (14). Neuroticism is closely related to depression as a stable and heritable personality trait (15). Depression was also commonly seen in patients seeking aesthetic therapies. For example, patients who received breast implants exhibited high rates of suicidal ideation (16). Conversely, in other patients, breast augmentation effectively improved one's self-assessment (17). The negative correlation between cosmetic improvement and the intention in surgery suggested that cosmetic treatment helps increase life satisfaction.

Personality is described as a way of perceiving and relating the environment to oneself, and is affected by both genetics and the acquired environment. Relevant aspects of personality exist in a wide range of contexts, and are relatively stable over time (18). According to Eysenck, personality encompasses three major dimensions. Extraversion refers to sociability, vivacity, enthusiasm, and impulsivity. Neuroticism epitomizes depression, anxiousness, and emotional instability. Psychoticism signifies solitude, coldness, aggressiveness, and egocentricity (19).
There exists an inadequate number of reports concerning the assessment of personality traits in patients prior to undergoing cosmetic procedures. Participants possessing psychopathological traits in the cosmetic industry were lower than expected, and their levels of anxiety did not cause dysfunction (20). Integral personality scales have rarely been applied to elucidate aesthetic orientation. How can we provide treatment options that match one's personality?

It is necessary to explore the association between personality profiles and the decision made in undergoing cosmetic treatment. Differences in personality lead to diverse choices, which are significant references for doctors and beauty seekers regarding recovery period, postoperative expectations, and design of the operation. Moreover, compared to the widely consumption beauty markets, cosmetology psychology in China is still in its infancy. In terms of plastic surgery, this study is the first to conduct personality questionnaires. It is hypothesized that different personality traits will influence the selection of treatment sites. This study may also assist in the clarification of psychological profiles, how they relate to an individuals' aesthetic tendency, and may contribute to optimal outcomes in therapy.

\section{MATERIALS AND METHODS}

\section{Participants}

This was an observational cross-sectional study conducted at the Second Affiliated Hospital of Zhejiang University between September 1 and December 6, 2019. The inclusion criteria were: (1) participants were capable of filling out questionnaires independently; (2) participants demonstrated physical fitness without evidence of deformities, scars, or severe systemic diseases. Participants completed a demographic selfquestionnaire with their name, gender, age, height, weight, income, marital status, education, smoking and drinking history, sleep duration, type of cosmetic treatment, and psychological scales. A total of 473 patients were investigated by random sampling. Finally, 426 questionnaires were included in the analysis, excluding questionnaires that rejected the survey, provided incomplete questionnaire information or untrue information.

The study was approved by the hospital Ethics Committee. All patients were informed of the objectives of the study, and they provided their informed consent.

We use the term "cosmetic treatment," which is inclusive of surgeries (e.g., blepharoplasty, rhinoplasty, and liposuction) and non-surgical treatments (e.g., Botulinum toxin or filler injections and lasers).

\section{Measures}

The EPQ (an 88-item self-reporting scale revised in China) was utilized in this study due to its excellent reliability and validity, which involves four scales: the extraversion $(\mathrm{E})$, neuroticism $(\mathrm{N})$, psychoticism (P) and lying scales (L). The higher the score, the more likely the personality traits listed in the scale are shown.

Based on the total score that each participant received in each scale, the standard score $\left[\mathrm{T}=50+10^{*}(\mathrm{X}-\mathrm{M}) / \mathrm{SD}\right]$ was obtained by conversion. $\mathrm{M}$ and $\mathrm{SD}$ refer to the mean 
and standard deviation of the original scores achieved by the normal groups, respectively. Depending on the levels of the internal and external propensity scales and the neuroticism scale, the participants in the study were split into four classic temperaments: sanguineous (extroverted, stable), choleric (extroverted, unstable), phlegmatic (introverted, stable), and melancholic (introverted, unstable) (19).

\section{Statistical Analysis}

Based on the statistics from 2000 (21), the average normal population in each dimension was calculated using the total scores of males and females in each dimension (number*mean) divided by the total number of participants. Participants who had or were predisposed to cosmetic treatment were considered the case group, with the remainder being the control group.

The data were input into Epidata 3.1 software, and Stata 15.1 was used for statistical analysis. All EPQ scores and demographic data were analyzed using descriptive statistics. Age, body mass index (BMI), sleep duration, and EPQ scores were regarded as continuous variables, whereas the cosmetic treatment tendency, results, and demographic parameters were considered categorical variables. The continuous variables were indicated by their mean and standard deviation (SD), and categorical variables were expressed as numbers $(N)$ and percentages (\%). A One-sample $t$-test was conducted to analyze the differences in EPQ scores at different sites. Moreover, a binary logistic regression analysis was conducted to investigate the correlation between EPQ scores at different sites and the demographic variables. $P<0.05$ was regarded as being statistically significant.

\section{RESULTS}

The general information of all participants is presented in Table 1. There were 426 eligible patients included in this study, which was comprised of 384 (90.14\%) females and 42 (9.86\%) males. The age of the sample group ranged from 17 to 64.5 years $($ Mean $=32.14, \mathrm{SD}=8.06)$. Additionally, $310(72.77 \%)$ participants possessed a bachelor's degree or higher, and 213 (50\%) were married. Over $60 \%$ of participants exhibited a BMI within the normal range.

Of the 426 participants, the preferred treatment sites were the skin, eyes (including eyebrows and lacrimal sulcus area), nose, face contour, and body (including breasts, abdomen, leg, shoulder, and labia minora). Cosmetic treatments involved laser rejuvenation, blepharoplasty, rhinoplasty, breast augmentation, chin augmentation, liposuction, botulinum toxin, or filler injections.

The mean and standard deviation values of the $\mathrm{E}$ score reached $11.48 \pm 3.34, \mathrm{P}$ score of $4.80 \pm 2.32, \mathrm{~N}$ score of 11.39 \pm 5.63 , and L score of $11.67 \pm 3.40$, as shown in Table 1 and Figure 1 indicates the EPQ scores for participants with or without cosmetic treatment according to treatment sites. In the general population, the average of $\mathrm{E}$ was 11.50 , while the averages of $\mathrm{P}, \mathrm{N}$, and $\mathrm{L}$ were 5.67, 10.88, and 12.56, respectively (21). Comparatively, three of the scales (E, P, and L) scored lower than the normal average, but $\mathrm{N}$ was higher than the normal average. As for L, lying signifies unsophisticated dissimulation,
TABLE 1 | General information of the participants included $(n=426)$.

\begin{tabular}{|c|c|}
\hline Characteristics & Means or proportions \\
\hline Age (years, mean \pm SD) & $32.14 \pm 8.06$ \\
\hline \multicolumn{2}{|l|}{ Gender, $\boldsymbol{n}(\%)$} \\
\hline Male & $42(9.86)$ \\
\hline Female & $384(90.14)$ \\
\hline \multicolumn{2}{|l|}{ Education, $\boldsymbol{n}(\%)$} \\
\hline Bachelor degree or below & $116(27.23)$ \\
\hline Bachelor degree or above & $310(72.77)$ \\
\hline \multicolumn{2}{|l|}{ BMI, $n(\%)$} \\
\hline$<18.5$ & $108(25.35)$ \\
\hline $18.5-24$ & $288(67.61)$ \\
\hline$\geq 24$ & $30(7.04)$ \\
\hline \multicolumn{2}{|l|}{ Marital, $\boldsymbol{n}(\%)$} \\
\hline Single & $213(50)$ \\
\hline Married & $213(50)$ \\
\hline \multicolumn{2}{|l|}{ Income, $n(\%)$} \\
\hline Stable & 128 (30.05) \\
\hline Unstable & 298 (69.95) \\
\hline \multicolumn{2}{|l|}{ Smoke, $n(\%)$} \\
\hline Current/ever & $26(6.10)$ \\
\hline Never & $400(93.90)$ \\
\hline \multicolumn{2}{|l|}{ Drink, $n(\%)$} \\
\hline Current/ever & $44(10.33)$ \\
\hline Never & 382 (89.67) \\
\hline \multicolumn{2}{|l|}{ Sleep duration, $n(\%)$} \\
\hline$<7 \mathrm{~h}$ & $51(11.97)$ \\
\hline $7-8 h$ & $174(40.85)$ \\
\hline$\geq 8 \mathrm{~h}$ & $201(47.18)$ \\
\hline \multicolumn{2}{|c|}{ Eysenck Personality Scale, mean \pm SD } \\
\hline E score & $11.48 \pm 3.34$ \\
\hline P score & $4.80 \pm 2.32$ \\
\hline N score & $11.39 \pm 5.63$ \\
\hline L score & $11.67 \pm 3.40$ \\
\hline \multicolumn{2}{|l|}{ Sites, $\boldsymbol{n}(\%)$} \\
\hline Skin & $189(44.37)$ \\
\hline Nose & $36(8.45)$ \\
\hline Face contour & 119 (27.93) \\
\hline Eye & $103(24.18)$ \\
\hline Body & $41(9.62)$ \\
\hline
\end{tabular}

E, extraversion; N, neuroticism; P, psychoticism; L, lying.

where participants scored lower than those in the normal population. A difference was found between participants with or without face contours in psychoticism ( $P$ for heterogeneity $=0.002)$. Moreover, there were significant differences regarding site location and neuroticism, such as in the eye $(P=0.045)$, face contour $(P=0.002)$, nose $(P<0.001)$, or skin $(P=0.004)$.

The association between the choice of cosmetic site and the EPQ scores for participants choosing to undergo treatment is depicted in Table 2. The model was adjusted with respect to age, gender, BMI, education, marital status, incomes, smoking and drinking history, and sleep duration. A statistically negative association was observed between skin treatment and $\mathrm{N}$ score 

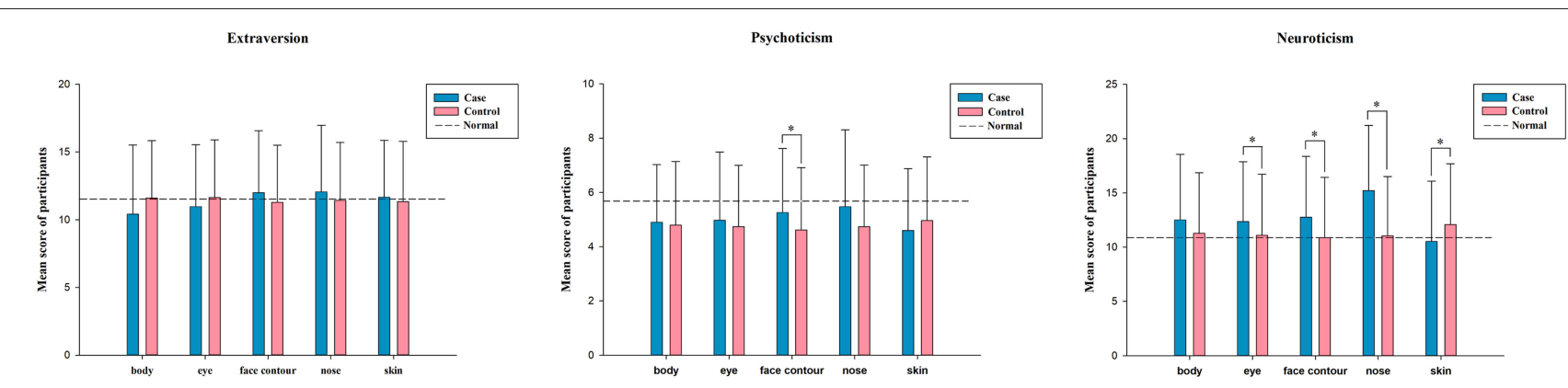

FIGURE 1 | Distribution of EPQ scores in participants with or without cosmetic treatment according to different sites (= $\bar{\chi} \pm \mathrm{SD}$ ). The case in the table refers to the participant who selects the site for cosmetic surgery, control refers to participants who have not selected the part for cosmetic surgery, normal refers to the average score of the general population in the scale. ${ }^{*}$ The results are statistically significant $(P<0.05)$.

TABLE 2 | Association between cosmetic surgery choice and EPQ scores in participants according to different sites $(n=426)$.

\begin{tabular}{|c|c|c|c|c|c|c|c|c|c|c|}
\hline & \multicolumn{2}{|l|}{ Skin } & \multicolumn{2}{|l|}{ Nose } & \multicolumn{2}{|c|}{ Face contour } & \multicolumn{2}{|l|}{ Eye } & \multicolumn{2}{|l|}{ Body } \\
\hline & OR $(95 \% \mathrm{Cl})$ & $P$ & OR (95\% Cl) & $P$ & OR (95\% Cl) & $P$ & OR (95\% Cl) & $P$ & OR (95\% Cl) & $P$ \\
\hline E score & $1.02(0.98,1.07)$ & 0.370 & $1.01(0.92,1.10)$ & 0.863 & $1.05(1.00,1.11)$ & 0.044 & $0.95(0.90,1.01)$ & 0.076 & $0.94(0.87,1.02)$ & 0.131 \\
\hline P score & $0.95(0.87,1.04)$ & 0.285 & $1.11(0.95,1.29)$ & 0.184 & $1.13(1.03,1.25)$ & 0.013 & $1.03(0.93,1.14)$ & 0.575 & $1.03(0.89,1.19)$ & 0.717 \\
\hline N score & $0.96(0.92,1.00)$ & 0.031 & $1.15(1.07,1.24)$ & $<0.001$ & $1.05(1.01,1.10)$ & 0.019 & $1.04(1.00,1.09)$ & 0.068 & $1.05(0.98,1.12)$ & 0.143 \\
\hline Phlegmatic & $0.89(0.19,4.24)$ & 0.888 & - & & - & & $2.40(0.50,11.46)$ & 0.272 & - & \\
\hline Melancholic & $0.76(0.46,1.27)$ & 0.301 & $1.62(0.68,3.87)$ & 0.276 & $0.88(0.51,1.55)$ & 0.668 & $1.26(0.71,2.24)$ & 0.432 & $2.49(1.21,5.12)$ & 0.013 \\
\hline Sanguineous & $1.60(0.80,3.20)$ & 0.186 & $1.03(0.28,3.78)$ & 0.961 & $0.67(0.27,1.62)$ & 0.371 & $0.55(0.22,1.39)$ & 0.206 & $0.88(0.25,3.08)$ & 0.840 \\
\hline Choleric & $1.02(0.66,1.57)$ & 0.924 & $0.74(0.34,1.61)$ & 0.445 & $1.40(0.85,2.32)$ & 0.185 & $0.95(0.57,1.57)$ & 0.846 & $0.53(0.27,1.05)$ & 0.067 \\
\hline
\end{tabular}

OR, odds ratio; Cl, confidence interval; E, extraversion; P, psychoticism; N, neuroticism.

Logistic regression adjusted for age, gender, BMI, education, marital status, income, smoking, drinking and sleep duration.

The values in bold mean that the results are statistically significant $(P<0.05)$.
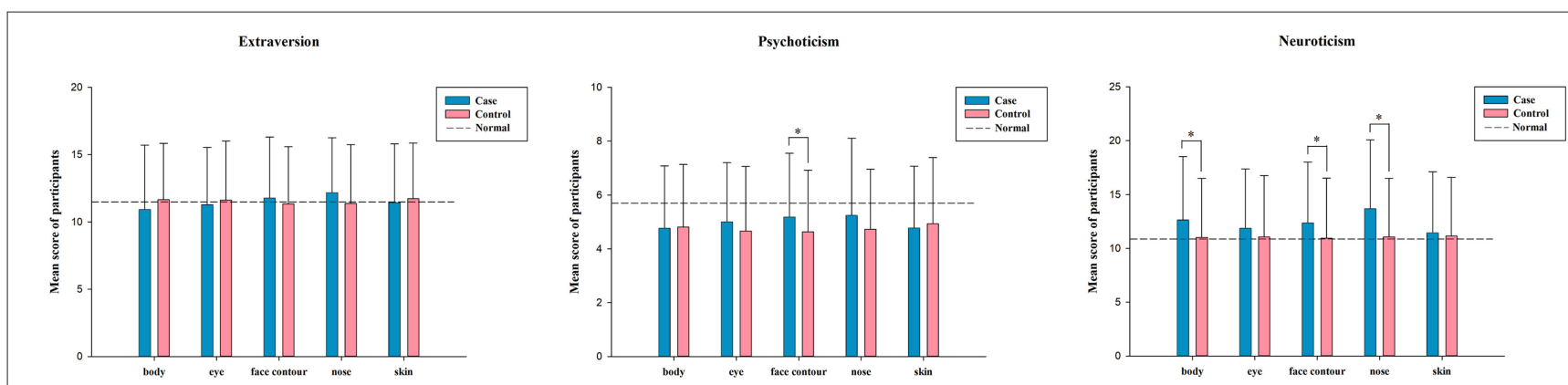

FIGURE 2 | Distribution of EPQ scores in participants with cosmetic treatment tendency according to different sites (= $\bar{\chi} \pm \mathrm{SD})$. The case in the table refers to the participant who prefers surgery on the site, control refers to participants who are not inclined to have surgery on that area, normal refers to the average score of the general population on the scale. *The results are statistically significant $(P<0.05)$.

risk (OR 0.96, 95\% CI $0.92-1.00, P=0.031$ ). Those in the skin treatment group, which serves as an option in conventional medical rejuvenation, demonstrated lower levels of anxiety. A significant positive association was observed between cosmetic treatments performed on nose and N score risk (OR 1.15, 95\% CI $1.07-1.24, P<0.001)$, and a positive association was identified between face contour treatment and $\mathrm{E}, \mathrm{P}$, and $\mathrm{N}$ scores (OR $1.05,95 \%$ CI $1.00-1.11, P=0.044$; OR 1.13, CI 1.03-1.25, $P=0.013$; OR 1.05 , CI $1.01-1.10, P=0.019$ ). In addition, regarding the four personality portraits, melancholic personality demonstrated a positive association with body treatment risk (OR 2.49, CI 1.21-5.12, $P=0.013$ ). However, other personality traits were not statistically significant with respect to the choice in treatment sites.

The distribution of EPQ scores for participants with a tendency to receive cosmetic treatment according to different sites on their body is illustrated in Figure 2. This tendency suggests that participants wanted to achieve 
TABLE 3 | Association between cosmetic surgery tendency and EPQ scores in participants according to different sites $(n=426)$.

\begin{tabular}{|c|c|c|c|c|c|c|c|c|c|c|}
\hline & \multicolumn{2}{|l|}{ Skin } & \multicolumn{2}{|l|}{ Nose } & \multicolumn{2}{|c|}{ Face contour } & \multicolumn{2}{|l|}{ Eye } & \multicolumn{2}{|l|}{ Body } \\
\hline & OR (95\% Cl) & $\boldsymbol{P}$ & OR (95\% Cl) & $P$ & OR (95\% Cl) & $P$ & OR (95\% CI) & $\boldsymbol{P}$ & OR (95\% CI) & $\boldsymbol{P}$ \\
\hline E score & $0.98(0.92,1.04)$ & 0.452 & $1.03(0.96,1.11)$ & 0.403 & $1.03(0.98,1.08)$ & 0.241 & $0.98(0.93,1.02)$ & 0.328 & $0.96(0.91,1.01)$ & 0.135 \\
\hline P score & $0.97(0.87,1.09)$ & 0.663 & $1.06(0.93,1.20)$ & 0.408 & $1.09(0.99,1.20)$ & 0.067 & $1.10(1.00,1.20)$ & 0.046 & $0.98(0.89,1.09)$ & 0.770 \\
\hline N score & $1.02(0.97,1.07)$ & 0.534 & $1.07(1.01,1.14)$ & 0.018 & $1.03(0.99,1.07)$ & 0.212 & $1.04(1.00,1.08)$ & 0.035 & $1.06(1.01,1.10)$ & 0.018 \\
\hline Phlegmatic & - & & $1.42(0.15,13.39)$ & 0.760 & $1.16(0.20,6.62)$ & 0.865 & $1.02(0.22,4.81)$ & 0.982 & $1.51(0.27,8.50)$ & 0.638 \\
\hline Melancholic & $1.23(0.62,2.44)$ & 0.562 & $0.58(0.25,1.39)$ & 0.224 & $0.92(0.53,1.58)$ & 0.761 & $1.15(0.70,1.91)$ & 0.578 & $1.46(0.83,2.55)$ & 0.188 \\
\hline Sanguineous & $0.87(0.36,2.13)$ & 0.766 & $1.43(0.51,4.03)$ & 0.499 & $0.84(0.37,1.90)$ & 0.670 & $0.60(0.29,1.23)$ & 0.161 & $0.65(0.26,1.63)$ & 0.356 \\
\hline Choleric & $0.81(0.45,1.44)$ & 0.470 & $1.23(0.62,2.47)$ & 0.554 & $1.12(0.70,1.81)$ & 0.634 & $1.09(0.71,1.69)$ & 0.687 & $0.83(0.50,1.37)$ & 0.473 \\
\hline
\end{tabular}

OR, odds ratio; Cl, confidence interval; E, extraversion; P, psychoticism; N, neuroticism.

Logistic regression adjusted for age, gender, BMI, education, marital status, income, smoking, drinking and sleep duration.

The values in bold mean that the results are statistically significant $(P<0.05)$.

cosmetic transformation, but had yet to undergo an operation. Statistics showed that neuroticism held the highest mean EQP score, while psychoticism had the lowest mean score for different sites. A difference was discovered between the groups which with or without face contour treatment tendencies in those with psychoticism $(P=0.022)$. Additionally, there were significant differences existing in patients with neuroticism regarding body $(P=0.010)$, face contour $(P=0.017)$, or nose $(P=0.001)$ cosmetic treatment tendencies.

As shown in Table 3, an association is evident between the cosmetic treatment tendency and EPQ scores according to various sites. In terms of treatment tendency of cosmetic, the participants who preferred cosmetic surgery on nose (OR 1.07, CI 1.01-1.14, $P=0.018$ ), eyes (OR 1.04, CI 1.00-1.08, $P=$ 0.035 ) or body (OR 1.06, CI 1.01-1.10, $P=0.018$ ) site had statistical difference in $\mathrm{N}$ score. Moreover, the tendency of eye surgery showed an association with the P score (OR 1.10, CI 1.00-1.20, $P=0.046)$. No significant differences were observed between the other scores and the four personality traits at different sites.

\section{DISCUSSION}

To our knowledge, this is the first study employing EPQ to assist with the psychometric analysis of cosmetic treatment. Participant profiles were predominantly young females with a high level of education, healthy lifestyle, and insecure incomes. These findings were consistent with Wei's study in regard to the general information (22). In their study, young women were the main patients, including some high school and college students Women were more dissatisfied with their body compared to men (23). With the change in aesthetics and the influence of social media, women pay more attention to their personal appearance, physical dissatisfaction may have a negative impact on the patient's self-image, social status, employability and interpersonal relationships (24). Health conditions and depression also participate in encouraging people to have cosmetic surgery $(25,26)$. Additionally, public perception of cosmetic surgery has also changed as more people have become educated (1).
Further studies have found that psychological factors, such as the pursuit of beauty and body satisfaction have a greater impact on cosmetic surgery patients than social factors, like having higher social standing and building good interpersonal relationships (27). We investigated the psychological characteristics to determine the treatment preferences of different groups in hopes of achieving improved treatment effects and outcomes.

The most popular surgical sites were the skin, face contour, and eyes in our study, which align with other findings (28). Patients $<20$-year-old were likely to choose minor interventions, such as skin treatment, while those over 45 preferred rejuvenation procedures (29). Moreover, as Chinese are more conservative regarding aesthetics, indications for surgery are also more conservative than in other countries (30), breast augmentation is less considered. In view of treatment choices, participants showed a distinguished personality profile. First, the inward- and outward-leaning personality types are the most prominent psychological types proposed by Carl Jung. This dimension relates to the intensity of excitability and inhibition of the central nervous system. Extroverts tend to have additional social needs, seek more social interaction and value their appearance to gain energy from their environment (31). Hence, they may be more motivated to attain beauty through medical procedures. Second, neuroticism was the most common psychological trait motivating patients to undergo cosmetic surgery on the nose, eyes, and other body parts (e.g., breast, legs and shoulders). Neuroticism may be represented by depression, anxiety, and emotional instability. Patients manifesting depressive symptoms often possess lower levels of self-esteem (32). They expect to achieve their desired body image and hope to reduce their anguish and dissatisfaction. Third, patients with a high degree of psychoticism scores have a potential psychopathological-associated personality trait. They are likely to feel lonely and ignore others, and they tend to exhibit peculiar behavior like suicide while disregarding danger (33). Therefore, when they consider undergoing plastic surgery, they may ignore the risks.

Among the patients who have undergone plastic surgery, neuroticism scores for the nose were the highest, which 
referred to its susceptibility for negative impacts, such as negative emotions (34). A high level of consistency was present where neurotic patients demonstrated interest in having nose treatments and followed through with receiving a rhinoplasty. According to Brucoli, rhinoseptoplasty patients are characterized by anxiety, depression, and less pronounced passivity, but exhibit higher levels of self-esteem (35). Patients who sought rhinoplasty for aesthetic motivations felt more depressed than those seeking functional rhinoplasty (36). In addition, obsessiveness and narcissism were detected in patients seeking rhinoplasty $(13,37)$. The human nose is considered the most prominent midline projection of the face (38), and is considered the most noticeable and concerning site regarding one's personal characteristics. Moreover, congenital defects or aesthetic needs of the nose may lead to dissatisfaction and negative long-term effects on selfesteem. Low levels of self-acceptance confer significant impacts on psychological resilience by causing anxiety and depression. This study confirmed the presence of a significant difference in the $\mathrm{N}$ score, which shows that the most attention was given to the nose. Patients who were satisfied with their rhinoplasty outcomes found that the procedure helped improve their body image and quality of life while boosting their self-confidence and self-esteem (39).

However, in those repeatedly dissatisfied with their surgical outcomes, consideration should be given to BDD patients (9). Identifying BPD patients or even those with psychotic personalities is necessary, as they may frequently demand treatments for multiple sites (11). They figuratively choose to attack the most prominent part of the face to alleviate the discomfort caused by their personality disorder. The EPQ questionnaire is limited to a screening questionnaire. Consideration can be given to the Adverse Childhood Experiences questionnaire and the Structured Interview of Personality Organization (STIPO) to carry out a more comprehensive screening.

Facial contour adjustment was indicative of extraversion, psychoticism, and neuroticism. Furthermore, the score of EPQ was higher than the control group. In previous studies, similar results were obtained. Participants with experience in masseter injections or mandibular therapies scored high in extraversion, agreeableness, openness, and neuroticism (40). These traits were often reflected in behavior rather than in psychoticism (37). Essentially, they resorted to cosmetic treatments due to a lack of confidence, which was associated with specific physical defects and the desire to socialize (41). In addition, the contour of the mandibular angle was significant to the facial shape of Asian women, who believe that women with wide and square faces were more likely to be unhappy (42). Therefore, these patients are obsessed with having oval shaped faces, smooth tapered jaws, and round, pointy chins following treatment (43).

In this study, participants who underwent treatment on certain body parts showed melancholic personality traits. Evidence showed that participants shifted from substantially alleviated depression following treatment, and from selfloathing $t$ to self-appreciation, illustrating an improved outlook on life (44). Certain depressed patients shown psychosomatic manifestations, and they improved their self-esteem and depressive symptoms following cosmetic surgery (45).

Regarding the tendencies for cosmetic treatment, a significant difference existed in the $\mathrm{N}$ score of the treatment of body sites, which is likely delayed in actuality. For example, liposuction or breast augmentation may carry high risks and a lengthy recovery. Interestingly, the human eye is another focus on the face, indicating the personality traits of neuroticism and psychoticism as well. However, the eyes can be refined by the use of cosmetics, glasses, and other modifications. Therefore, no significant difference was observed in our final results.

Our study contains several limitations. First, the majority of participants were located in east China, which may cause selection bias. Second, male data $(n=42)$ was inadequate in comparison with that of females. Third, the EPQ selfreporting scale is the most commonly conducted personality test in China due to its convenient implementation. It reflects a relatively limited range of personality types, making it difficult to conduct a more precise personality assessment. The different dimensions of the personality questionnaire should be further expanded, such as the Cattell Sixteen Personality Factor or the Big Five Personality Inventory. Despite these limitations, this study's findings highlight the impact of personality traits on different sites of cosmetic surgeries. In the future, postoperative satisfaction should be assessed, and more screening procedures should be designed for clinical intervention to avoid unnecessary surgeries.

\section{CONCLUSION}

The personality profile of participants receiving cosmetic treatment was more depression, anxiety, emotional instability and non-sophisticated. Different personality traits influence the site selection for cosmetic therapy. Physicians should consider neuroticism in patients seeking rhinoplasty. If experienced surgeons identify personality traits prior to undergoing cosmetic surgery, patients may benefit from better rehabilitation and increased satisfaction.

\section{DATA AVAILABILITY STATEMENT}

The raw data supporting the conclusions of this article will be made available by the authors, without undue reservation.

\section{ETHICS STATEMENT}

The studies involving human participants were reviewed and approved by the Human and Research Ethics committees of the Second Hospital of Zhejiang University. Written informed consent to participate in this study was provided by the participants' legal guardian/next of kin. Written informed consent was obtained from the individual(s), and minor(s)' legal guardian/next of kin, for the publication 
of any potentially identifiable images or data included in this article.

\section{AUTHOR CONTRIBUTIONS}

AS and MZhe: conceptualization and methodology. HQ: writing- original draft preparation. YL: formal analysis. MZha: data curation. CW: investigation. CL: review and editing. All authors contributed to the article and approved the submitted version.

\section{REFERENCES}

1. Haas CF, Champion A, Secor D. Motivating factors for seeking cosmetic surgery: a synthesis of the literature. Plast Surg Nurs. (2008) 28:17782. doi: 10.1097/PSN.0b013e31818ea832

2. Breuning EE, Oikonomou D, Singh P, Rai JK, Mendonca DA. Cosmetic surgery in the NHS: applying local and national guidelines. J Plast Reconstr Aesthet Surg. (2010) 63:1437-42. doi: 10.1016/j.bjps.2009.08.012

3. The American Society for Aesthetic Plastic Surgery. The American Society for Aesthetic Plastic Surgery's Cosmetic Surgery National Data Bank: Statistics 2016. Aesthet Surg J. (2016) 39:1-27.

4. American Society for Aesthetic Plastic Surgery. The American Society for Aesthetic Plastic Surgery's Cosmetic Surgery National Data Bank: Statistics 2018. Aesthet Surg J. (2019) 39(Suppl_4):1-27. doi: 10.1093/asj/sjz164

5. Qing ke Research Center. Retrieved from: www.pedata.cn (accessed April 14, 2021).

6. Spataro EA, Kandathil CK, Saltychev M, Olds CE, Most SP. Correlation of the standardized cosmesis health nasal outcomes survey with psychiatric screening tools. Aesthet Surg J. (2020) 40:1373-80. doi: 10.1093/asj/sjaa004

7. Shridharani SM, Magarakis M, Manson PN, Rodriguez ED. Psychology of plastic and reconstructive surgery: a systematic clinical review. Plast Reconstr Surg. (2010) 126:2243-51. doi: 10.1097/PRS.0b013e3181f445ae

8. Kuhn H, Cunha PR, Matthews NH, Kroumpouzos G. Body dysmorphic disorder in the cosmetic practice. Ital Dermatol Venereol. (2018) 153:506-15.

9. Wang Q, Cao C, Guo R, Li X, Lu L, Wang W, et al. Avoiding psychological pitfalls in aesthetic medical procedures. Aesthetic Plast Surg. (2016) 40:95461. doi: 10.1007/s00266-016-0715-9

10. Lieb K, Zanarini MC, Schmahl C, Linehan MM, Bohus M. Borderline personality disorder. Lancet. (2004) 364:45361. doi: 10.1016/S0140-6736(04)16770-6

11. Morioka D, Ohkubo F. Borderline personality disorder and aesthetic plastic surgery. Aesthetic Plast Surg. (2014) 38:116976. doi: 10.1007/s00266-014-0396-1

12. Lilienfeld SO. Longitudinal studies of personality disorders: four lessons from personality psychology. J Pers Disord. (2005) 19:547-56; discussion 594-46. doi: 10.1521/pedi.2005.19.5.547

13. Zojaji R, Arshadi HR, Keshavarz M, Mazloum Farsibaf M, Golzari F, Khorashadizadeh M. Personality characteristics of patients seeking cosmetic rhinoplasty. Aesthetic Plast Surg. (2014) 38:1090-3. doi: 10.1007/s00266-014-0402-7

14. Parsa KM, Gao W, Lally J, Davison SP, Reilly MJ. Evaluation of personality perception in men before and after facial cosmetic surgery. JAMA Facial Plast Surg. (2019) 21:369-74. doi: 10.1001/jamafacial.2019.0463

15. Li JJ, Hilton EC, Lu Q, Hong J, Greenberg JS, Mailick MR. Validating psychosocial pathways of risk between neuroticism and late life depression using a polygenic score approach. J Abnorm Psychol. (2019) 128:20011. doi: $10.1037 / \mathrm{abn} 0000419$

16. de Paula PR, Fortes de Arruda FC, Prado M, Neves CG. Prevalence of depressive symptoms in patients requesting cosmetic breast surgery in midwestern Brazil. Plast Reconstr Surg Glob Open. (2018) 6:e1899. doi: 10.1097/GOX.0000000000001899

17. Rzepa T, Grzesiak W, Zaborski D, Modrzejewski A, Pastucha M. Physical attractiveness and self-assessment before and after breast augmentation.

\section{FUNDING}

This work was funded by Zhejiang Provincial Natural Science Foundation of China (LGD21H150001).

\section{ACKNOWLEDGMENTS}

We acknowledge the contribution of participating patients and medical students for data collection. Furthermore, we gratefully thank Dr. Li Yingjun for contribution in statistics.
Ann Plast Surg. (2014) 72:618-24. doi: 10.1097/SAP.0b013e3182 6aeffe

18. Battle DE. Diagnostic and statistical manual of mental disorders (DSM). Codas. (2013) 25:191-2. doi: 10.1590/s2317-17822013000200017

19. Gong L, Dong JY. Patient's personality predicts recovery after total knee arthroplasty: a retrospective study. J Orthop Sci. (2014) 19:2639. doi: 10.1007/s00776-013-0505-Z

20. Del Aguila E, Martinez JR, Pablos JL, Huanuco M, Encina VM, Rhenals AL. Personality traits, anxiety, and self-esteem in patients seeking cosmetic surgery in Mexico City. Plast Reconstr Surg Glob Open. (2019) 7:e2381. doi: 10.1097/GOX.0000000000002381

21. Lyu Y. Study on the Reliability and Validity of the Second Eysenck Personality Questionnaire (master). Changsha: Cnki; Central South University (in Chinese) (2008).

22. Wei L, Ge C, Xiao W, Zhang X, Xu J. Cross-sectional investigation and analysis of anxiety and depression in preoperative patients in the outpatient department of aesthetic plastic surgery in a general hospital in China. J Plast Reconstr Aesthet Surg. (2018) 71:1539-46. doi: 10.1016/j.bjps.2018.07.015

23. Quittkat HL, Hartmann AS, Dusing R, Buhlmann U, Vocks S. Body dissatisfaction, importance of appearance, and body appreciation in men and women over the lifespan. Front Psychiatry. (2019) 10:864. doi: 10.3389/fpsyt.2019.00864

24. Hekmatpanah J. Evidence-based treatment of cavernous sinus meningioma. Surg Neurol Int. (2019) 10:228. doi: 10.25259/SNI_268_2019

25. Nerini A, Matera C, Stefanile C. Psychosocial predictors in consideration of cosmetic surgery among women. Aesthetic Plast Surg. (2014) 38:4616. doi: 10.1007/s00266-014-0294-6

26. Cheraghian B, Fereidooni-Moghadam M, Babadi H, Dashtbozorgi B. Psychological and personality characteristics of applicants for facial cosmetic surgery. Aesthetic Plast Surg. (2020) 44:780-7. doi: 10.1007/s00266-02001682-6

27. Babadi H, Fereidooni-Moghadam M, Dashtbozorgi B, Cheraghian B. Investigating psychosocial causes of the tendency for facial cosmetic surgery. Aesthetic Plast Surg. (2018) 42:1157-63. doi: 10.1007/s00266-018-1078-1

28. Javo IM, Sorlie T. Psychosocial characteristics of young Norwegian women interested in liposuction, breast augmentation, rhinoplasty, and abdominoplasty: a population-based study. Plast Reconstr Surg. (2010) 125:1536-43. doi: 10.1097/PRS.0b013e3181d5135a

29. Rini IS, Krisna MA, Basuki A, Djarot KR. The characteristics of private plastic surgery practice in developing country: an epidemiological study. Indian J Plast Surg. (2018) 51:309-15. doi: 10.4103/ijps.IJPS_11_18

30. Luo CX, Wen ZH, Zhen Y, Wang ZJ, Mu JX, Zhu M, et al. Chinese research into severe ulcerative colitis has increased in quantity and complexity. World J Clin Cases. (2018) 6:35-43. doi: 10.12998/wjcc.v6.i3.35

31. Rodriguez-Enriquez M, Bennasar-Veny M, Leiva A, Yanez AM. Alcohol and tobacco consumption, personality, and cybervictimization among adolescents. Int J Environ Res Public Health. (2019) 16:3123. doi: 10.3390/ijerph16173123

32. Sertoz OO, Doganavsargil O, Elbi H. Body image and selfesteem in somatizing patients. Psychiatry Clin Neurosci. (2009) 63:508-15. doi: 10.1111/j.1440-1819.2009.01994.x

33. Fonseca-Pedrero E, Ortuno-Sierra J, Inchausti F, Rodriguez-Testal JF, Debbane M. Beyond clinical high-risk state for psychosis: the network 
structure of multidimensional psychosis liability in adolescents. Front Psychiatry. (2019) 10:967. doi: 10.3389/fpsyt.2019.00967

34. Klamer S, Schwarz L, Kruger O, Koch K, Erb M, Scheffler K, et al. Association between neuroticism and emotional face processing. Sci Rep. (2017) 7:17669. doi: 10.1038/s41598-017-17706-2

35. Brucoli M, Baena RRY, Boffano P, Benech A. Psychological profiles in patients undergoing orthognathic surgery or rhinoplasty: a preoperative and preliminary comparison. Oral Maxillofac Surg. (2019) 23:179-86. doi: 10.1007/s10006-019-00758-1

36. Naraghi M, Atari M. A comparison of depression scores between aesthetic and functional rhinoplasty patients. Asian J Psychiatr. (2015) 14:2830. doi: 10.1016/j.ajp.2015.01.009

37. Zojaji R, Javanbakht M, Ghanadan A, Hosien H, Sadeghi H. High prevalence of personality abnormalities in patients seeking rhinoplasty. Otolaryngol Head Neck Surg. (2007) 137:83-7. doi: 10.1016/j.otohns.2007.02.027

38. Wright MR. The psychology of rhinoplasty. Facial Plast Surg. (1988) 5:10913. doi: 10.1055/s-2008-1064743

39. Borujeni LA, Pourmotabed S, Abdoli Z, Ghaderi H, Mahmoodnia L, Sedehi $\mathrm{M}$, et al. A comparative analysis of patients' quality of life, body image and self-confidence before and after aesthetic rhinoplasty surgery. Aesthetic Plast Surg. (2020) 44:483-90. doi: 10.1007/s00266-019-01559-3

40. Scharschmidt D, Mirastschijski U, Preiss S, Brahler E, Fischer T, Borkenhagen A. Body image, personality traits, and quality of life in botulinum toxin A and dermal filler patients. Aesthetic Plast Surg. (2018) 42:111925. doi: 10.1007/s00266-018-1165-3

41. Meningaud JP, Benadiba L, Servant JM, Herve C, Bertrand JC, Pelicier Y. Depression, anxiety and quality of life: outcome 9 months after facial cosmetic surgery. J Craniomaxillofac Surg. (2003) 31:46-50. doi: 10.1016/S1010-5182(02)00159-2

42. Kim SK, Han JJ, Kim JT. Classification and treatment of prominent mandibular angle. Aesthetic Plast Surg. (2001) 25:382-7. doi: $10.1007 / \mathrm{s} 002660010150$

43. Samizadeh $\mathrm{S}, \mathrm{Wu} \mathrm{W}$. Ideals of facial beauty amongst the Chinese population: results from a Large National Survey. Aesthetic Plast Surg. (2018) 42:1540-50. doi: 10.1007/s00266-018$1188-9$

44. Gilmartin J, Long AF, Soldin M. Changing body image and well-being: following the experience of massive weight loss and body contouring surgery. Healthcare. (2014) 2:150-65. doi: 10.3390/healthcare2020150

45. Golshani S, Mani A, Toubaei S, Farnia V, Sepehry AA, Alikhani M. Personality and psychological aspects of cosmetic surgery. Aesthetic Plast Surg. (2016) 40:38-47. doi: 10.1007/s00266-015-0592-7

Conflict of Interest: The authors declare that the research was conducted in the absence of any commercial or financial relationships that could be construed as a potential conflict of interest.

Copyright $\odot 2021$ Qian, Ling, Wang, Lenahan, Zhang, Zheng and Shao. This is an open-access article distributed under the terms of the Creative Commons Attribution License (CC BY). The use, distribution or reproduction in other forums is permitted, provided the original author(s) and the copyright owner(s) are credited and that the original publication in this journal is cited, in accordance with accepted academic practice. No use, distribution or reproduction is permitted which does not comply with these terms. 